\title{
Self-report of current and prior antiretroviral drug use in comparison to the medical record among HIV-infected patients receiving primary HIV care
}

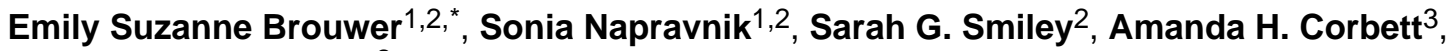 \\ and Joseph J. Eron Jr.2 \\ ${ }^{1}$ Department of Epidemiology, University of North Carolina, Chapel Hill, NC, USA \\ 2 Department of Medicine, University of North Carolina, Chapel Hill, NC, USA \\ ${ }^{3}$ Department of Pharmacy, University of North Carolina, Chapel Hill, NC, USA
}

\section{Abstract}

Objective-Patient antiretroviral (ARV) therapy knowledge is essential for regimen adherence, successful therapeutic response, and minimization of resistance evolution. Moreover, a complete and accurate patient ARV history is needed to construct efficacious and tolerable future regimens. In this study we assessed the ability of HIV-infected patients receiving care in a university infectious diseases clinic to accurately recall current and past ARVs.

Methods-A convenience sample $(n=205)$ of UNC HIV Clinical Cohort participants $(n=1840)$ completed a comprehensive in-person interview. Patients were asked about current and ever ARV use and were provided proprietary and generic ARV names and photographs. Self-reported sensitivity for current and ever ARV use (proportion that correctly identified all recorded ARVs), was calculated using the medical record as the gold standard.

Results-One hundred and eighty-five patients had received ARVs at some point after enrollment in the cohort study (ever users). For current ARV use $(n=138)$, self-reported sensitivity was $63 \%$ (95\% CI: $54-71)$. For ever use $(n=185)$, sensitivity was $18 \%$ (95\% CI: $13-$ 24).

Conclusion-Self-reported cumulative ARV use is not accurate. Since HIV-infected patients are prescribed a number of medications over their treatment course, it is necessary to develop new medication reconciliation techniques that are not dependent on patient memory or knowledge in order to improve patient outcomes.

\section{Keywords}

HIV infection; antiretroviral therapy; patient education; patient non-adherence

Copyright (C) 2011 John Wiley \& Sons, Ltd.

${ }^{*}$ Correspondence to: E. S. Brouwer, Gillings School of Global Public Health, Department of Epidemiology, University of North Carolina, Chapel Hill, NC, USA ebrouwer@email.unc.edu.

CONFLICT OF INTEREST

ESB was supported by an unrestricted fellowship from the UNC-GlaxoSmithKline Center of Excellence in Pharmacoepidemiology and Public Health at the University of North Carolina, Gillings School of Global Public Health while conducting this research. GlaxoSmithKline markets or has marketed antiretroviral medications. JJE has been a paid consultant to Merck, Pfizer, GlaxoSmithKline, Tibotec and Bristol MyersSquibb. In addition, he has received honoraria from Gilead. Each of these companies market or have marketed antiretroviral medications. None of these companies have provided support for this research. All other authors, no known conflicts. 


\section{INTRODUCTION}

Combinations of currently approved antiretroviral (ARV) medications can effectively suppress HIV viral replication, even in the presence of multidrug resistant variants. ${ }^{1-3}$ Although the durability of an individual patient's first ARV regimen is increasing, ${ }^{4,5}$ patients switch ARVs for a multitude of reasons, including toxicity and virologic failure. In one study, almost one-fifth of patients received their fourth ARV regimen after just 4 years from initiating therapy, ${ }^{6}$ and as many as $10-20 \%$ of HIV-infected patients in care may harbor ARV drug resistance to all three commonly used ARV drug classes. ${ }^{7,8}$ Since virologic failure and ARV drug mutations are strongly related to progression to an AIDS defining clinical event and death, ${ }^{8}$ increasing the durability of each ARV regimen is a critical clinical goal.

One of the primary components of successful sustained virologic response to ARV therapy is maintaining adequate adherence. ${ }^{9-13}$ ARV therapy adherence is associated with a number of patient socio-demographic, behavioral, and clinical factors, including ARV knowledge. ${ }^{9,14-16}$ Although ARV knowledge may affect clinical outcomes, in part through its effect on adherence, little is known about patient knowledge of the ARVs they are currently receiving or those they have received in the past. ${ }^{17}$ Successful treatment of patients who have had previous therapy and virologic rebound is also dependent on selection of the most active, well tolerated regimen for an individual patient. ${ }^{18}$ Resistance testing provides important information, however, accurate treatment histories may be equally important as a current resistance test may only reveal mutations selected by the patient's current regimen. In addition, patients may present off therapy when resistance testing is less helpful. Knowledge of ARV tolerability also impacts regimen selection. For all these reasons, an accurate ARV treatment history is extremely important. Furthermore, as HIV patients are often transient, visiting many different providers over their treatment course, patient report may be a clinician's primary source for ARV treatment history.

Studies investigating patient knowledge of non-ARV medication use has suggested that many patients may have trouble recalling the medications they are taking. ${ }^{19-22}$ Therefore, we designed this study to assess the sensitivity and accuracy of patient self-report of ARV use, including current and prior use. We also assessed whether any patient demographic or clinical characteristics were associated with improved ARV self-report.

\section{METHODS}

\section{Study population}

A convenience sample $(n=205)$ of UNC CFAR HIV Clinical Cohort study participants $(n=$ 1840) were approached by clinic staff to also complete an in-person interview between 1999 and 2006, with only two individuals completing the interview in 1999 and the remainder completing the interview after January 2001. The 177 item ongoing interview addresses topics outside of the patients' normal clinical care and surveys patients on clinical, behavioral and socio-demographic matters related to their HIV infection. On specific interview days, study staff approached all patients who were consented to participate in the UNC CFAR HIV Clinical Cohort study and gave permission for study staff to approach them for additional research activities. Interviews were conducted on all days of the week so as to interview patients seen by all providers. No exclusion criteria were used to select patients who completed the interview. All patients receiving HIV care, irrespective of whether or not they were receiving antiretroviral therapy, who provided informed consent to participate in the UNC CFAR HIV Clinical Cohort were eligible to complete the interview. Patients who completed the interview were representative of the overall cohort. The UNC CFAR HIV Clinical Cohort study enrolls HIV-1-infected patients receiving primary HIV 
care at a large tertiary medical center in the Southeastern United States. ${ }^{23}$ Cohort data consists of all institutionally available electronic patient records and periodic standardized medical record reviews, including detailed ARV data.

\section{Study measures}

As part of the in-person interview, patient self-reported ARV use was assessed relying on two separate questions, one for "current use" and one for "ever use". To facilitate recall, proprietary and generic names accompanied by photographs of the medications were provided. Interview administrators did not provide any additional information to the patient pertaining to ARV use (e.g., dosing, administration requirements, or side effects). The interview instrument was updated over time to account for the availability of new ARVs. The medical record was considered the 'Gold Standard'. Ever ARV use was defined as any use prior to and including the interview date. Current use was defined as use on the date of the interview. A correct identification of a fixed dose combination (FDC) medication included the patient naming either the FDC or each drug entity contained within the FDC. For example, a patient who self-reports zidovudine and lamivudine would be classified as having correct recall for the drug entity Combivir ${ }^{\circledR}$ (a combination of zidovudine and lamivudine). Similarly, a patient who self-reports Combivir ${ }^{\circledR}$ would be classified as having correct recall if the medical record indicated that they were taking zidovudine and lamivudine.

\section{Statistical analysis}

ARV self-report and medical record agreement was assessed by comparing patient responses to the medical record. Perfect ARV self-report was defined as identifying all ARVs recorded in the clinical record (self-report sensitivity). We performed separate analyses to assess selfreport sensitivity for the ARVs a patient was currently taking and all the ARVs a patient had ever received. We evaluated whether patient demographic (e.g., sex, age, race, and education level), clinical (e.g., CD4 cell count, HIV RNA level, and cumulative number of ARVs), and/or study characteristics (year of interview) were associated with ARV selfreport sensitivity. All current and prior ARVs prescribed were used to calculate the cumulative number of ARVs per patient with FDCs considered one ARV. Chi-square and Fisher's exact tests were used to contrast proportions and the Wilcoxon rank-sum and Kruskal-Wallis tests were used for continuous variables.

We also assessed the proportion of ARVs a patient correctly named (self-report percent accuracy), calculated as the number of self-reported ARVs also in the clinical record divided by the number of ARVs in the clinical record. This was done for both the patients' current ARV regimen and their cumulative ARV exposure. We evaluated whether patient demographic, clinical, and study characteristics were associated with self-report accuracy. The Kruskal-Wallis and the Wilcoxon rank-sum tests were used to examine differences among groups. To evaluate the correlation between current and ever percent accuracy, we calculated the Pearson correlation coefficient $(r)$ and calculated the associated confidence interval using Fisher's transformation.

Finally, we evaluated whether ARV self-report and medical record agreement were related to adherence. Adherence was measured by patient self-report of missed doses within the previous 3 days as well as the ability to maintain a medication schedule: always, most of the time, sometimes, rarely or never. The adherence assessment instrument is a modification of the Adult AIDS Clinical Trials Group adherence assessment as previously described. ${ }^{24}$ The instrument was modified using input from clinical staff and other study personnel. 
This study was approved by the Institutional Review Board of The University of North Carolina at Chapel Hill and all patients included in this study provided written informed consent to participate. All statistical analyses were conducted using the SAS statistical package (version 9.1; SAS Institute, Inc., Cary, NC, USA).

\section{RESULTS}

\section{Study population}

The participants $(n=205)$ were predominantly black $(71 \%)$, male $(66 \%)$, and a median age of 42 years [interquartile range (IQR): 36-47; Table 1]. Approximately one-third of the study sample did not complete high school. At the time of the interview, patients had been in care for an average of 6.7 years, almost one-half had HIV-1 RNA below 400 copies $/ \mathrm{ml}$, and the median CD4 cell count was 407 cells $/ \mathrm{mm}^{3}$. Compared to the overall cohort, the study population had a greater proportion of African-American patients $(p=0.006)$. Nadir CD4 cell count levels were slightly higher in the study population $(p=0.08)$, while the peak HIV RNA values were comparable to the overall cohort $(p=0.98)$. HIV transmission risk behaviors in the study sample were similar to the overall cohort.

Twenty (10\%) patients were ARV-naïve and an additional 47 (23\%) were not taking ARVs at the time of the interview. Overall patients had received a median of five ARVs (IQR, 2-7; full range, 0-19) over the course of their ARV treatment. At the time of the interview nearly all patients receiving ARVs were taking a nucleoside(tide) reverse transcriptase inhibitor (98\%), two-thirds (65\%) a protease inhibitor, and 37\% a non-nucleoside reverse transcriptase inhibitor.

\section{Antiretroviral self-report and medical record agreement}

Among the 138 patients taking ARVs at the time of the interview, 87 could name all the ARVs they were receiving (self-report sensitivity: 63\%; 95\% CI: 54-71). Of the 67 patients not taking any ARVs according to their medical record, $21 \%(n=14)$ reported taking at least one ARV (95\% CI: 12-33). Self-report sensitivity decreased substantially when we considered all previously received ARVs. Among the 185 patients who had ever received ARVs, 33 reported all the ARVs they had ever received for a self-report sensitivity of $18 \%$ (95\% CI: 13-24). Of the 20 patients who had never received any ARVs, $30 \%(n=6)$ reported receiving at least one ARV (95\% CI: 12-54).

The only factor that was statistically significantly related to self-report sensitivity of a current ARV regimen was patient sex. Women were more likely than their male counterparts to correctly report their current ARV medications (77\% vs. 54\%; $p$-value 0.02 ; Table 2). In analyses assessing ARV ever use, the number of cumulative ARVs received was strongly associated with self-report sensitivity. Specifically, $37 \%$ of patients who had received a maximum of four ARVs ever, were able to correctly name all the ARVs they had received; however, only $2 \%$ of patients who had received more than four ARVs in their lifetime could correctly name all the ARVs they had received ( $p$-value $<0.001)$.

On average, patients were able to name $75 \%$ of the ARVs they were currently taking and $43 \%$ of the ARVs they had ever received. The cumulative distribution of the self-report percent accuracy measure was skewed for both cumulative and current use. The majority of the patients had high self-report percent accuracy for current regimens, and low percent accuracy for their cumulative regimens. Further, as the number of prior ARVs a patient received increased, current and cumulative percent accuracy decreased (Figure 1). The Pearson's correlation coefficient calculated to examine the correlation between patients' current and cumulative percent accuracy suggests a positive relationship; as percent accuracy increased for current regimens, the percent accuracy for cumulative regimens also 
increased $(r=0.46,95 \%$ CI: $0.32-0.58)$. We did not identify any statistically significant associations with percent accuracy for current or cumulative ARV use (Table 3). However, while not statistically significant, the mean percent accuracy for current ARV regimens did increase over calendar time.

In a sensitivity analysis, we restricted the population to patients who initiated HIV care at the University of North Carolina Infectious Diseases Clinic. Forty-five percent $(n=92)$ of patients did not receive any prior HIV care. The current and cumulative self-report sensitivity for this sub-group of individuals was 66\% (95\% CI: 53-78\%) and 20\% (95\% CI: $11-30 \%)$, respectively. On average, these patients were able to accurately name $75 \%$ of their current regimens and $42 \%$ of their cumulative regimens.

\section{Antiretroviral use self-report and adherence}

Of the patients who provided a response to the number of doses missed within the last 3 days $(n=170), 83 \%$ reported zero-missed doses. Forty-seven percent $(n=94)$ of patients reported being able to keep on a regular schedule for taking HIV medications always, $42 \%$ $(n=85)$ most or some of the time, and 3\% $(n=5)$ rarely or never. Ten percent of patients $(n$ $=21$ ) did not provide a response to this question. Although not statistically significant, there was a suggestion that patients who were always able to keep on a regular medication schedule were more likely to be able to identify all current ARVs than patients who were not always or never able to keep on a regular medication schedule (OR: 1.75, 95\% CI: 0.79 3.86). Conversely, patients who were always able to keep on a regular medication schedule were less likely to be able to correctly identify all cumulative ARVs (OR: $0.68,95 \%$ CI: $0.31-1.45)$.

\section{DISCUSSION}

Our results demonstrate that many HIV-infected patients are unable to recall their current ARV regimen completely and accurately and also are unable to recall ARVs taken in past regimens. This is particularly concerning since a complete knowledge of previous ARV medication regimens a patient has received is essential for informing which ARVs and regimens a patient may respond to and tolerate in the future.

Previous studies of patient recall accuracy demonstrated sensitivities between $44 \%$ and $93 \%$; however, these studies focused on classes of medications such as 3-hydroxy-3methylglutaryl-coenzyme A (HMG-CoA) reductase inhibitors, anti-hypertensives, nonsteroidal anti-inflammatory agents, and hormone replacement therapy and not on individual medications. ${ }^{19-22}$ Our sensitivities of $63 \%$ and $18 \%$ suggest that HIV-infected patients may have more difficulty successfully recalling ARVs than patients taking other classes of medications such as anti-hypertensives and hormone replacement therapies, which tend to be single drug regimens.

We found that the number of ARVs a patient receives or has received in the past influences their ability to accurately recall ARVs prescribed. This observation is expected as each new drug added to the treatment armamentarium leads to additional complexity in an individual regimen, generating more information that an HIV patient must remember. This is particularly important as the number of available ARVs and ARV classes continues to increase over time. It is likely that our findings are generalizable to other patients and clinical conditions. Polypharmacy is common in treatment of the elderly and of other disease states, ${ }^{25-28}$ and the inability to accurately recall current medications or a medication history is likely a problem in these patient populations as well. 
Previous studies among HIV-positive patients have found that patients with low health literacy and little HIV therapy knowledge are less likely to accurately identify ARV medications. ${ }^{14,15,29,30}$ Furthermore, medication knowledge affects a patient's ability to adhere to medications. For example, Miller et al..$^{15}$ demonstrated that a better medication knowledge score was significantly associated with better adherence to ARVs at 8 weeks. However, this was not seen at weeks 24 and 48 suggesting that knowledge is just one of many factors that influence patients' adherence to ARVs. ${ }^{15}$ While we did find that patients who maintained a regular medication schedule were more likely to recall their current ARVs and less likely to recall their cumulative ARVs, this association was not statistically significant. Reasons for the observed paradoxical relationship between success at maintaining a medication schedule could be related to behaviors of patients and the design of the interview. Patients who are able to recall all their medications may be more honest about their ability to maintain a schedule and report more difficulty whereas patients who are unable to recall their medications may have greater perceived self-efficacy. We also did not demonstrate a statistically significant association between recall and current HIV RNA level (another marker of adherence). The presence of neurocognitive impairment in conjunction with the complexity and cumbersome nature of medication regimens to treat HIV may lead to difficulty recalling historical medication regimens, further supporting the multi-faceted relationship between knowledge of HIV, recall of ARVs, and adherence. For these reasons there is a need for additional medication reconciliation techniques targeted to all patients who do not rely on patient memory or knowledge of HIV disease.

Our study has a few limitations. We considered the medical record as the gold standard, which is neither a perfect measure of prescription history nor does it indicate if a patient actually received or took the medications prescribed. ${ }^{31,32}$ Since the medical record may not be completely accurate for patients who transferred HIV care from another facility we also conducted analyses restricted to patients who initiated ART at our site. In these analyses, we did not find a meaningful difference between patients followed entirely at UNC infectious diseases clinic compared to the complete study population. Our study is also subject to recall bias as a patient who experienced an untoward side effect to a medication (e.g., abacavir hypersensitivity) may be more likely to recall that medication over another, resulting in imperfect exchangeability of each ARV. However, we designed our instrument to assist the participants with recall, by including specific ARV drug names (proprietary and generic) and providing photographs of each ARV. ${ }^{33,34}$ Finally, the selection of patients to complete the in-person interview was not conducted at random. Nevertheless, all patients seen in the clinic that were consented to participate in the UNC CFAR HIV Clinical Cohort study were approached on each selected interview day. Despite the limitations, this assessment highlights the importance of interventions designed to enhance a patient's awareness of their medications, which will eventually lead to improved outcomes.

Novel tools to assist in medication reconciliation are necessary in order to reduce dependency on patient factors, such as memory and knowledge. These instruments can be provided to patients to maintain a personal record of ARVs and concomitant medications. At the time of this study, the use of medication charts by HIV-positive patients was less common than currently. Their use has continued to increase, however, a more consistent use of these forms with the assistance of technology is needed. Making medication lists quickly modifiable by providers and/or patients, and providing the lists in a format that is easily maintained is important. One example is the use of USB storage devices that can be placed on patient's key chain, in a pocket, or in a purse and updated at each clinic visit. Another option is a web-based personal health record (PHR). This personally controlled health record could be modified by providers and patients. In this model the PHR is not institutionally based, but compatible with multiple medical records systems. This way a patient can keep information from multiple providers, over time or at various institutions, in one place and 
directly control sharing of information when necessary. While there are early projects exploring the use of PHRs, there are challenges in implementation to be worked out and much more evaluation necessary before they will be widely available. ${ }^{35}$ For those patients who prefer, a paper copy of their medications in a pocket size format would be a step-wise approach to the electronic format. Pharmacists and other providers in the clinic could work to ensure each patient has an accurate medication history with updates for patients at each visit. The use of these strategies would reduce the dependency on patient recall for medication history allowing for a more accurate representation of this information in the medical record. Interventions aimed at testing the utility of these tools within the HIV population are needed.

In summary, this study highlights the poor reliability of patient recall in reconstructing HIV medication histories. These findings indicate a need to implement a more consistent and reliable method of determining medication histories as this information is critical to providing safe and effective treatment of HIV.

\section{Acknowledgments}

This project was supported in part by grants M01RR00046 and UL1RR025747 from the National Center of Research Resources, National Institutes of Health. The project was also supported by National Institutes of Health grant P30 AI590410 and the Agency for Healthcare Research and Quality grant R01 HS018731. ESB was supported by an unrestricted fellowship from the UNC-GlaxoSmithKline Center of Excellence in Pharmacoepidemiology and Public Health at the University of North Carolina, Gillings School of Global Public Health.

\section{References}

1. Braitstein P, Brinkhof MW, Dabis F, et al. Mortality of HIV-1-infected patients in the first year of antiretroviral therapy: comparison between low-income and high-income countries. Lancet. 2006; 367(9513):817-824. [PubMed: 16530575]

2. Clotet B, Bellos N, Molina JM, et al. Efficacy and safety of darunavir-ritonavir at week 48 in treatment-experienced patients with HIV-1 infection in POWER 1 and 2: a pooled subgroup analysis of data from two randomised trials. Lancet. 2007; 369(9568):1169-1178. [PubMed: 17416261]

3. Madruga JV, Cahn P, Grinsztejn B, et al. Efficacy and safety of TMC125 (etravirine) in treatmentexperienced HIV-1-infected patients in DUET-1: 24-week results from a randomised, double-blind, placebo-controlled trial. Lancet. 2007; 370(9581):29-38. [PubMed: 17617270]

4. Moore RD, Keruly JC, Gebo KA, et al. An improvement in virologic response to highly active antiretroviral therapy in clinical practice from 1996 through 2002. J Acquir Immune Defic Syndr. 2005; 39(2):195-198. [PubMed: 15905736]

5. Willig JH, Abroms S, Westfall AO, et al. Increased regimen durability in the era of once-daily fixed-dose combination antiretroviral therapy. AIDS. 2008; 22(15):1951-1960. [PubMed: 18784459]

6. Chen RY, Westfall AO, Mugavero MJ, et al. Duration of highly active antiretroviral therapy regimens. Clin Infect Dis. 2003; 37(5):714-722. [PubMed: 12942406]

7. Napravnik S, Keys JR, Quinlivan EB, et al. Triple-class antiretroviral drug resistance: risk and predictors among HIV-1-infected patients. AIDS. 2007; 21(7):825-834. [PubMed: 17415037]

8. Cozzi-Lepri A, Phillips AN, Clotet B, et al. Detection of HIV drug resistance during antiretroviral treatment and clinical progression in a large European cohort study. AIDS. 2008; 22(16):21872198. [PubMed: 18832882]

9. Parruti G, Manzoli L, Toro PM, et al. Long-term adherence to first-line highly active antiretroviral therapy in a hospital-based cohort: predictors and impact on virologic response and relapse. AIDS Patient Care STDS. 2006; 20(1):48-56. [PubMed: 16426156]

10. Simoni JM, Pearson CR, Pantalone DW, et al. Efficacy of interventions in improving highly active antiretroviral therapy adherence and HIV-1 RNA viral load. A meta-analytic review of 
randomized controlled trials. J Acquir Immune Defic Syndr. 2006; 43 (Suppl 1):S23-35. [PubMed: 17133201]

11. Liu H, Miller LG, Hays RD, et al. Repeated measures longitudinal analyses of HIV virologic response as a function of percent adherence, dose timing, genotypic sensitivity, and other factors. J Acquir Immune Defic Syndr. 2006; 41(3):315-322. [PubMed: 16540932]

12. Gross R, Yip B, Lo Re V III, et al. A simple, dynamic measure of antiretroviral therapy adherence predicts failure to maintain HIV-1 suppression. J Infect Dis. 2006; 194(8):1108-1114. [PubMed: 16991085]

13. Harrigan PR, Hogg RS, Dong WW, et al. Predictors of HIV drug-resistance mutations in a large antiretroviral-naive cohort initiating triple antiretroviral therapy. J Infect Dis. 2005; 191(3):339347. [PubMed: 15633092]

14. Wolf MS, Davis TC, Arozullah A, et al. Relation between literacy and HIV treatment knowledge among patients on HAART regimens. AIDS Care. 2005; 17(7):863-873. [PubMed: 16120503]

15. Miller LG, Liu H, Hays RD, et al. Knowledge of antiretroviral regimen dosing and adherence: a longitudinal study. Clin Infect Dis. 2003; 36(4):514-518. [PubMed: 12567311]

16. Mills EJ, Nachega JB, Bangsberg DR, et al. Adherence to HAART: a systematic review of developed and developing nation patient-reported barriers and facilitators. PLoS Med. 2006; 3(11):e438. [PubMed: 17121449]

17. Wood E, Kerr T, Hogg RS, et al. Validity of self-reported antiretroviral therapy use among injection drug users. J Acquir Immune Defic Syndr. 2006; 41(4):530-531. [PubMed: 16652065]

18. Hammer SM, Eron JJ Jr, Reiss P, et al. Antiretroviral treatment of adult HIV infection: 2008 recommendations of the International AIDS Society-USA Panel. JAMA. 2008; 300(5):555-570. [PubMed: 18677028]

19. Boudreau DM, Daling JR, Malone KE, et al. A validation study of patient interview data and pharmacy records for antihypertensive, statin, and antidepressant medication use among older women. Am J Epidemiol. 2004; 159(3):308-317. [PubMed: 14742292]

20. Sjahid SI, van der Linden PD, Stricker BH. Agreement between the pharmacy medication history and patient interview for cardiovascular drugs: the Rotterdam elderly study. Br J Clin Pharmacol. 1998; 45(6):591-595. [PubMed: 9663815]

21. Rosenberg MJ, Layde PM, Ory HW, et al. Agreement between women's histories of oral contraceptive use and physician records. Int J Epidemiol. 1983; 12(1):84-87. [PubMed: 6302013]

22. West SL, Strom BL, Freundlich B, et al. Completeness of prescription recording in outpatient medical records from a health maintenance organization. J Clin Epidemiol. 1994; 47(2):165-171. [PubMed: 8113825]

23. Napravnik S, Eron JJ Jr, McKaig RG, et al. Factors associated with fewer visits for HIV primary care at a tertiary care center in the Southeastern U. S. AIDS Care. 2006; 18 (Suppl 1):S45-S50. [PubMed: 16938674]

24. Chesney MA, Ickovics JR, Chambers DB, et al. Self-reported adherence to antiretroviral medications among participants in HIV clinical trials: the AACTG adherence instruments. Patient Care Committee and Adherence Working Group of the Outcomes Committee of the Adult AIDS Clinical Trials Group (AACTG). AIDS Care. 2000; 12(3):255-266. [PubMed: 10928201]

25. Linjakumpu T, Hartikainen S, Klaukka T, et al. Use of medications and polypharmacy are increasing among the elderly. J Clin Epidemiol. 2002; 55(8):809-817. [PubMed: 12384196]

26. Jorgensen T, Johansson S, Kennerfalk A, et al. Prescription drug use, diagnoses, and healthcare utilization among the elderly. Ann Pharmacother. 2001; 35(9):1004-1009. [PubMed: 11573845]

27. Dailey G, Kim MS, Lian JF. Patient compliance and persistence with anti-hyperglycemic therapy: evaluation of a population of type 2 diabetic patients. J Int Med Res. 2002; 30(1):71-79. [PubMed: 11921502]

28. Veehof L, Stewart R, Haaijer-Ruskamp F, et al. The development of polypharmacy. A longitudinal study. Fam Pract. 2000; 17(3):261-267. [PubMed: 10846147]

29. Wolf MS, Davis TC, Osborn CY, et al. Literacy, self-efficacy, and HIV medication adherence. Patient Educ Couns. 2007; 65(2):253-260. [PubMed: 17118617]

30. Kalichman SC, Ramachandran B, Catz S. Adherence to combination antiretroviral therapies in HIV patients of low health literacy. J Gen Intern Med. 1999; 14(5):267-273. [PubMed: 10337035] 
31. Wagner MM, Hogan WR. The accuracy of medication data in an outpatient electronic medical record. J Am Med Inform Assoc. 1996; 3(3):234-244. [PubMed: 8723614]

32. Manley HJ, Drayer DK, McClaran M, et al. Drug record discrepancies in an outpatient electronic medical record: frequency, type, and potential impact on patient care at a hemodialysis center. Pharmacotherapy. 2003; 23(2):231-239. [PubMed: 12596688]

33. Klungel $\mathrm{OH}$, de Boer A, Paes AH, et al. Influence of correction for within-person variability in blood pressure on the prevalence, awareness, treatment, and control of hypertension. Am J Hypertens. 2000; 13(1 Pt 1):88-91. [PubMed: 10678276]

34. Gama H, Correia S, Lunet N. Questionnaire design and the recall of pharmacological treatments: a systematic review. Pharmacoepidemiol Drug Saf. 2009; 18(3):175-187. [PubMed: 19116956]

35. Halamka JD, Mandl KD, Tang PC. Early experiences with personal health records. J Am Med Inform Assoc. 2008; 15(1):1-7. [PubMed: 17947615] 


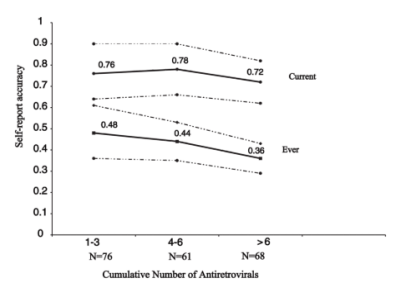

Figure 1.

Population mean (solid line) and corresponding 95\% confidence intervals (dashed line) of self-reported accuracy (number of correct self-reported ARVs also in the clinical record divided by the number of ARVs in the clinical record) by number of ARVs patients were taking at the time of the interview (current) and all ARVs recorded in the medical record at the time of the interview (ever) 


\section{Table 1}

Patient demographic and clinical characteristics for the study population and the UNC-CFAR HIV Clinical Cohort

\begin{tabular}{|c|c|c|}
\hline Characteristics & Study population $(n=205)$ & Cohort $(n=1840)$ \\
\hline Female, $n(\%)$ & $70(34)$ & $582(32)$ \\
\hline African-American, $n(\%)$ & $144(70)$ & $1094(59)$ \\
\hline Injection drug use, $n(\%)$ & $42(20)$ & $278(15)$ \\
\hline Men who have sex with men, $n(\%)$ & $77(38)$ & $674(36)$ \\
\hline Peak HIV RNA $\log _{10}$ (copies/ml), median (IQR) & $5.0(4.4-5.9)$ & $5.0(4.4-5.6)$ \\
\hline Nadir CD4 cell count (cells/ $\mu \mathrm{l})$, median (IQR) & $159(37-325)$ & $134(28-288)$ \\
\hline \multicolumn{3}{|l|}{ Patient characteristics at the time of interview } \\
\hline Age, median (IQR) & $42(36-47)$ & N/A \\
\hline Education $<12$ years, $n(\%)$ & $73(36)$ & N/A \\
\hline Current HIV RNA <400 copies/ml, $n(\%)$ & $98(48)$ & N/A \\
\hline Current CD4 cell count (cells/ $\mu \mathrm{l})$, median (IQR) & $407(228-620)$ & N/A \\
\hline Number of prior ARVs, median (IQR) & $5(2-7)$ & N/A \\
\hline Years in HIV care, median (IQR) & $6.7(3.9-9.5)$ & N/A \\
\hline
\end{tabular}

ARV, antiretroviral; IQR, interquartile range; N/A, not available/applicable. 


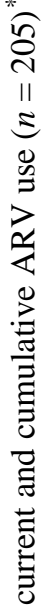




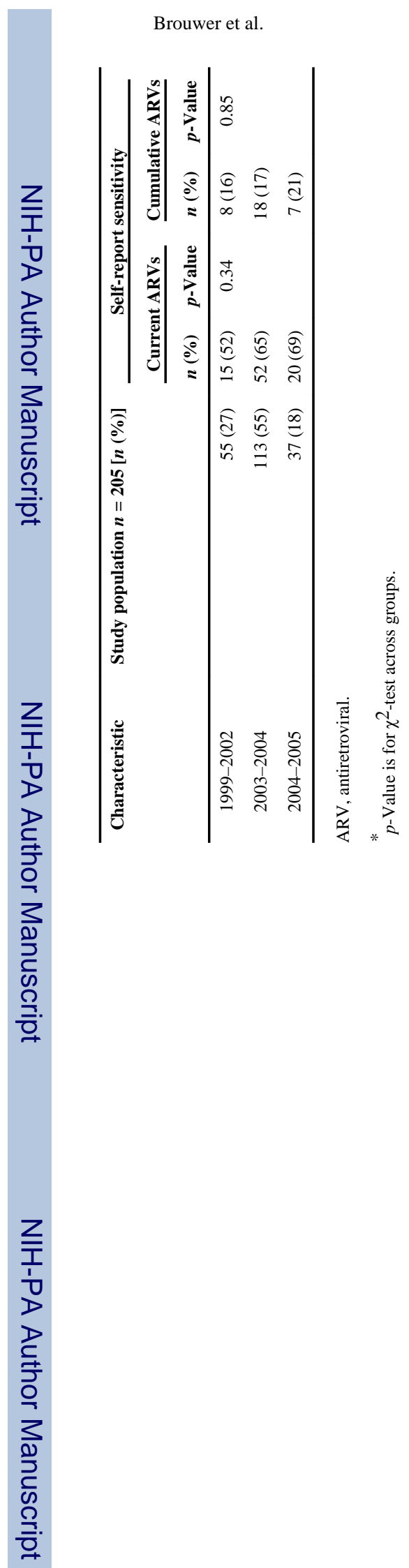

Pharmacoepidemiol Drug Saf. Author manuscript; available in PMC 2012 April 1. 


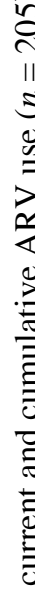




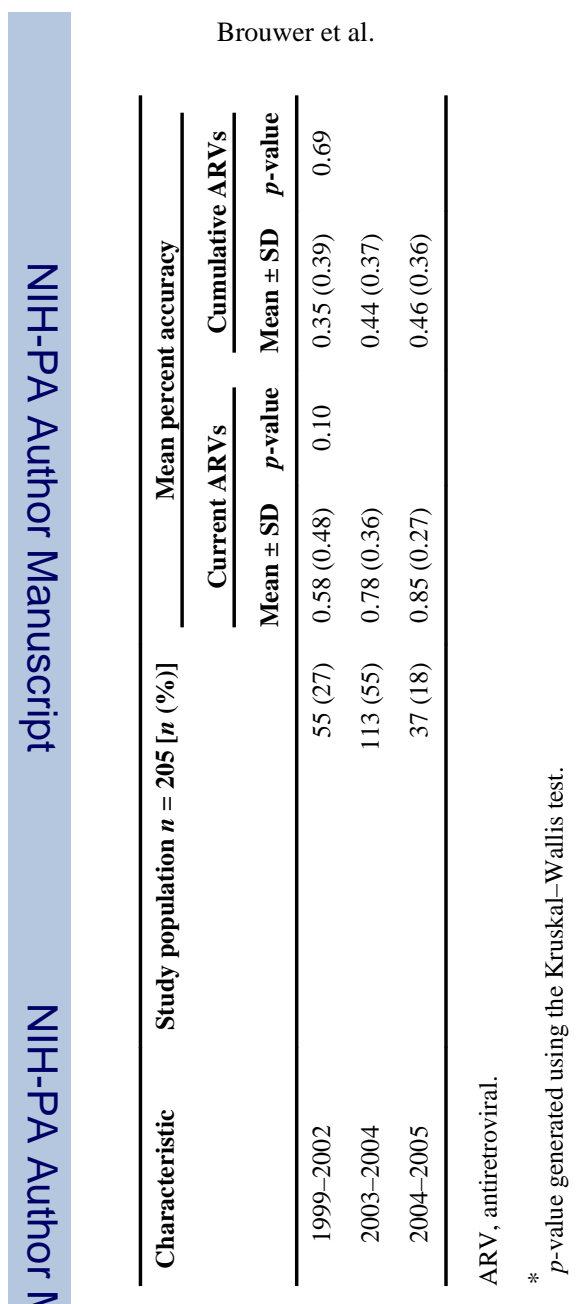

Pharmacoepidemiol Drug Saf. Author manuscript; available in PMC 2012 April 1. 